Lucía Ortega Donaire

1. Graduada en Enfermería. Máster en Gerontología Social: Iongevidad, salud y calidad Profesora sustituta interina, Universidad de Jaén. Doctoranda por la Universidad de Jaén, Jaén, España.

Correo electrónico: luciaod91@gmail.com

Recibido el 26 de agosto de 2019; aceptado el 10 de octubre de 2019

\section{Calidad de vida en personas mayores con síndrome de apnea obstructiva del sueño. Revisión sistemática}

\section{Quality of life in older people with obstructive sleep apnea syndrome. Systematic review}

\section{RESUMEN}

Objetivos: Identificar cómo afecta a la calidad de vida de las personas de edad avanzada padecer apnea obstructiva del sueño. Metodología: Revisión sistemática sobre los diferentes estudios que traten la apnea del sueńo en personas de edad avanzada, y cómo afecta esta a su calidad de vida. Esta revisión incluye casos y controles, estudios de cohortes, ensayos clínicos y estudios observacionales publicados desde 2014 hasta 2019. Resultados: Se revisaron cerca de 400 artículos,

de los cuales solo 16 formaron parte de la revisión, tras cumplir con exactitud todos los criterios de inclusión establecidos. Existe una gran disparidad entre los resultados encontrados; aun así, la calidad de vida de las personas de edad avanzada con apnea obstructiva del sueńo mejora con el uso de la CPAP (presión positiva continua en la vía aérea). Conclusiones: Son necesarias más investigaciones que estudien el síndrome de apnea obstructiva del sueño en personas de edad avanzada; la mayoría de los estudios encontrados se centran en población infantil o adulta. Entre los pocos estudios que se centran en estudiar la calidad de vida de las personas de edad avanzada cuando padecen apnea obstructiva del sueńo se encuentra disparidad en sus resultados, no llegando a ninguna conclusión firme. Este es un tema prioritario de salud, debido al envejecimiento de la población y a la cada vez mayor prevalencia de este síndrome.

PALABRAS CLAVE: Calidad de vida; persona de edad avanzada; persona de edad avanzada, 80 ańos en adelante; apnea obstructiva del sueńo.

\section{ABSTRACT}

Aims: To identify how obstructive sleep apnea affects the quality of life of the elderly. Methods: Systematic review of the different studies that treat sleep apnea in the elderly, and how it affects their quality of life. This review includes cases and controls, cohort studies, clinical trials and observational studies published from 2014 to 2019. Results: Nearly 400 articles were reviewed, of which only 16 were part of the review, after accurately meeting all established inclusion criteria. There is a large disparity between the results found; however, the quality of life of older people with obstructive sleep apnea improves with the use of CPAP (continuous positive air pressure). Conclusions: More research is needed to study obstructive sleep apnea syndrome in the elderly; most of the studies found focus on children or adults. Among the few studies that focus on studying the quality of life of older people with obstructive sleep apnea, there is a disparity in their results, with no firm conclusions. This is a priority health issue, due to the ageing population and the increasing prevalence of this syndrome.

KEYWORDS: Quality of life; aged; aged, 80 and over; sleep, apnea obstructive.

\section{- INTRODUCCIÓN}

Históricamente, el término síndrome de apnea del sueño se remonta al ańo 330 a. C, en la antigua Grecia, donde se aludía a este síndrome en un texto, refiriéndose al Rey Ponto como una persona obesa, glotona y con somnolencia diurna. Ya en el siglo xix, Charles Dickens, en su novela "Los papeles del Club Pickwick", habla sobre los trastornos relacionados con la obesidad y la hipoventilación asociada. Es en 1972 cuando Christian Guillemineault empieza a utilizar el término "síndrome de apnea del sueńo", aunque este término no se incorpora hasta la década de los 80 del pasado siglo, cuando Collin Sullivan habla de la presión positiva continua en la vía aérea como tratamiento del mismo $^{1}$. En la actualidad, el síndrome de apnea obstructiva del sueńo (SAOS), también conocido como síndrome de apnea-hipopnea del sue- ño (SAHS), es definido por el Consenso Nacional sobre el síndrome de apneas-hipopneas del sueño como: "la aparición de episodios recurrentes de limitación al paso del aire durante el sueño, como consecuencia de una alteración anatómico-funcional de la vía aérea superior (VAS) que conduce al colapso, provocando descensos de la saturación de oxihemoglobina $\left(\mathrm{SaO}_{2}\right)$ y microdespertares que dan lugar a un sueño no reparador, somnolencia diurna excesiva, trastornos neuropsiquiátricos, respiratorios y cardiacos"2.

El SAOS es un síndrome de alta prevalencia en la población general, la cual puede originar deterioro de la calidad de vida de las personas, así como empeoramiento de otras comorbilidades presentes en el organismo como son la hipertensión arterial y enfermedades cardiovasculares y cerebrovasculares, entre otras. La apnea obstructiva del sueño se relaciona con un exceso de mortalidad (calidad de evidencia alta) ${ }^{3}$. 
Los pacientes que no están diagnosticados duplican el consumo de los recursos sanitarios respecto a las personas que sí están diagnosticadas y tratadas. En España, entre el 3\% y el 6\% de la población padece apnea obstructiva del sueño sintomática y entre el $24 \%$ y el $26 \%$ padece apnea del sueño en su grado más elevado ${ }^{1,3}$.

En lo que respecta a los factores de riesgo a la hora de padecer esta enfermedad, encontramos: edad, sexo masculino e índice de masa corporal entre los más relevantes. La edad, a pesar de ser uno de los factores de riesgo más importantes, es el menos estudiado. En la actualidad, se desconoce si el mecanismo por el que se produce este colapso de la vía aérea en los ancianos se debe al propio envejecimiento o a una verdadera situación patológica; también encontramos como desconocido el verdadero punto de corte para poder diagnosticar apnea del sueńo en la población anciana o escalas adaptadas a este grupo de población. Esto es un problema de especial importancia, dada la cada vez mayor longevidad de la población. En España sufren apnea obstructiva del sueño el triple de ancianos que personas de mediana edad, por lo que este trastorno del sueńo se considera un problema de relevancia epidemiológica que afecta tanto a la salud de este grupo de población y a su calidad de vida como a la población que los rodea. A pesar de ello, este es un problema infradiagnosticado y subtratado en el grupo de población anciana ${ }^{3}$.

\section{- OBJETIVOS}

- Identificar cómo afecta a la calidad de vida de las personas de edad avanzada padecer apnea obstructiva del sueño.

- Conocer cuáles son los tratamientos utilizados en la actualidad para mejorar la calidad de vida de las personas de edad avanzada con apnea obstructiva del sueño.

\section{- METODOLOGÍA}

\section{Tipo de estudio}

Revisión sistemática de estudios de investigación realizados sobre apnea obstructiva del sueño en población anciana y calidad de vida.

\section{Método de búsqueda}

La búsqueda de estudios publicados se realizó en las principales bases de datos bibliográficas: PubMed, CUIDEN, Cochrane, LILACS, Cuidatge, IBECS, SciELO, Scopus y Google Scholar, con publicaciones desde 2014 hasta 2019. Como descriptores se han utilizado los términos: "Quality of life" o "sleep apnea obstructive" y "elderly" (tabla 1). Adicionalmente, se ha realizado una búsqueda bibliográfica inversa a partir de las referencias bibliográficas de los estudios ya seleccionados.

Para la pregunta de investigación, se utilizó el formato $\mathrm{PICO}^{4}:$ ¿Cómo es la calidad de vida de personas mayores de 65 años con apnea obstructiva del sueño?

\section{Criterios de inclusión y exclusión}

Los criterios de inclusión utilizados para realizar esta revisión han sido:

1. Publicación del artículo con una antigüedad inferior a 5 años (20142019).

2. Tener acceso al texto completo.

3. Estar en español, inglés o catalán.
Tabla 1. Bases de datos consultadas y palabras clave/cadenas de búsqueda utilizadas

\begin{tabular}{|c|c|}
\hline Base de datos & Palabra clave/cadena de búsqueda \\
\hline PubMed & $\begin{array}{l}\text { (((Sleep apnea, obstructive [mh] OR Apnea[tiab] } \\
\text { OR Respiration disorders[tiab] OR Sleep apnea } \\
\text { syndrome*[tiab])) AND (Aged[mi] OR Aged, } 80 \text { and over[m]] } \\
\text { OR Elderly[tiab])) AND Quality of life[mi] }\end{array}$ \\
\hline CUIDEN & $\begin{array}{l}\text { ("calidad")AND((“de")AND((“vida")AND(("apnea") } \\
\text { AND(("obstructiva")AND((“"del")AND(("sueño") } \\
\text { AND("ancianos")|)|)) }\end{array}$ \\
\hline Cochrane & Quality of life AND elderly AND sleep apnea obstructive \\
\hline LILACS & Quality of life AND elderly AND sleep apnea obstructive \\
\hline Cuidatge & Quality of life AND elderly AND sleep apnea obstructive \\
\hline IBECS & $\begin{array}{l}\text { Quality AND of AND life AND elderly AND sleep AND } \\
\text { apnea AND obstructive }\end{array}$ \\
\hline SciELO & Quality of life AND elderly AND sleep apnea obstructive \\
\hline Scopus & Quality of life AND elderly AND sleep apnea obstructive \\
\hline Google Scholar & Quality of life AND elderly AND sleep apnea obstructive \\
\hline
\end{tabular}

4. Tener relación directa con los objetivos que se pretende conseguir en la revisión bibliográfica.

Se han excluido todos aquellos que no cumplían los criterios de inclusión establecidos, junto con los que tuvieran algún tipo de coste para poder acceder a ellos o se centraran en grupos de población diferentes a los ancianos.

\section{Extracción de datos}

De cada estudio incluido se extrajeron los siguientes datos:

- Datos generales: autores y año de publicación, duración del estudio, tipo de estudio, tamaño de la muestra, grupos que forman el caso si procede y principales resultados.

- Datos sobre efectividad clínica: alteración de la calidad de vida de las personas de edad avanzada con apnea obstructiva del sueño.

\section{Síntesis de datos}

Dada la imposibilidad de realizar una agregación matemática de los datos por la disparidad o heterogeneidad de métodos utilizados en los diferentes estudios, se presenta una agregación narrativa de los datos.

\section{- RESULTADOS}

Los resultados de la búsqueda se presentan en la figura 1 .

\section{Resultados generales de los estudios encontrados}

De los 16 estudios utilizados en esta revisión bibliográfica, 3 son revisiones sistemáticas ${ }^{5-7}$. En la tabla 2 se presentan las principales carac- 


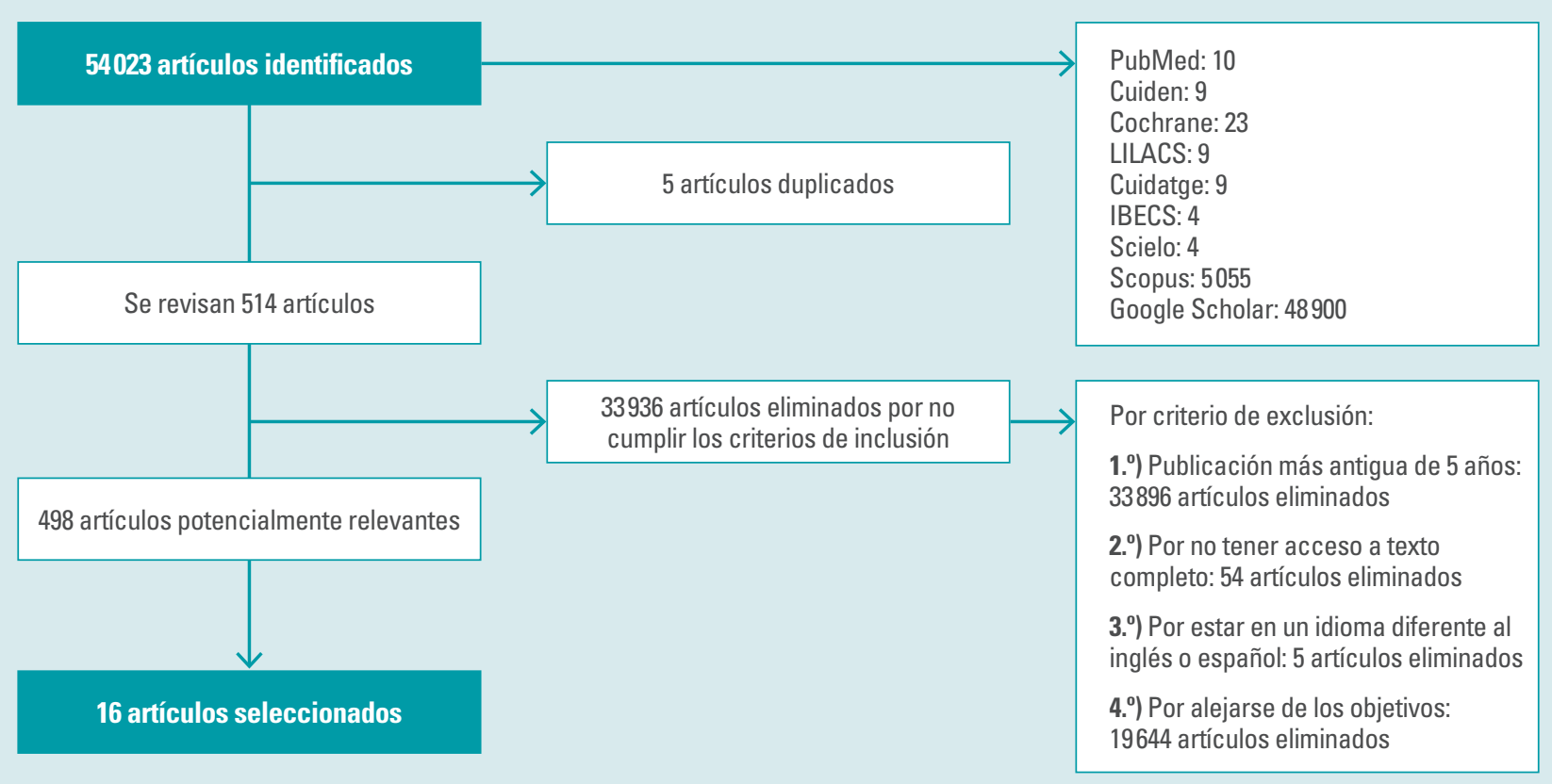

Figura 1. Diagrama de flujo de los estudios identificados.

Tabla 2. Principales características de los estudios incluidos

\begin{tabular}{|c|c|c|c|}
\hline Autor, año & $\begin{array}{l}\text { Duración } \\
\text { del estudio }\end{array}$ & $\begin{array}{l}\text { Tipo de estudio } \\
\text { Muestra (grupos) }\end{array}$ & Resultados \\
\hline $\begin{array}{l}\text { Lima TAG, et al., } \\
2019^{16}\end{array}$ & - & $\begin{array}{l}\text { Estudio transversal } \\
\qquad n=72\end{array}$ & No existe relación entre calidad de vida y apnea obstructiva del sueño \\
\hline $\begin{array}{l}\text { Serrano Merino J, } \\
\text { et al., } 2018^{8}\end{array}$ & 3 meses & $\begin{array}{l}\text { Estudio prospectivo } \\
\qquad n=103\end{array}$ & $\begin{array}{l}\text { El uso de CPAP mejora la calidad de vida de las personas de edad } \\
\text { avanzada con apnea del sueño }\end{array}$ \\
\hline $\begin{array}{l}\text { Appleton SL, et } \\
\text { al., } 2015^{17}\end{array}$ & 2 años & $\begin{array}{l}\text { Estudio de cohorte } \\
\qquad n=1869\end{array}$ & $\begin{array}{l}\text { Hombres } \leq 69 \text { años: disminución significativa en calidad de vida, en el } \\
\text { dominio vitalidad, función física y salud general } \\
\text { Hombres } \geq 70 \text { años: no se encuentran alteraciones asociadas }\end{array}$ \\
\hline $\begin{array}{l}\text { Dalmases M, et } \\
\text { al., } 2016^{9}\end{array}$ & 3 meses & $\begin{array}{c}\text { Estudio de casos y controles } \\
\begin{array}{c}n=30 \\
\text { G1: uso de CPAP } \\
n=15 \\
\text { G2: no uso de CPAP } \\
n=15\end{array}\end{array}$ & $\begin{array}{l}\text { G1: mejora en calidad de vida y estado de ánimo } \\
\text { G2: no existen diferencias significativas }\end{array}$ \\
\hline $\begin{array}{l}\text { Martínez-García } \\
\text { MA, et al., } 2015^{10}\end{array}$ & 3 meses & $\begin{array}{l}\text { Ensayo clínico abierto, aleatorizado, multicéntrico } \\
\qquad \begin{array}{c}\text { en cohorte clínica } \\
n=224 \text { AOS grave } \\
\text { G1: } \\
n=115 \text { usan CPAP } \\
\text { G2: } \\
n=109 \text { sin uso de CPAP }\end{array}\end{array}$ & $\begin{array}{l}\text { G1: uso de CPAP } \\
\text { Mejoría en la calidad de vida en todos los dominios } \\
\text { G2: No CPAP } \\
\text { No diferencias significativas }\end{array}$ \\
\hline $\begin{array}{l}\text { Petrousky DV, et } \\
\text { al., } 2018^{6}\end{array}$ & - & $\begin{array}{l}\text { Revisión sistemática } \\
6 \text { estudios son analizados }\end{array}$ & $\begin{array}{l}\text { La interrupción del sueño se asocia de forma negativa a la calidad de } \\
\text { vida en sus cuatro dominios en personas con demencia }\end{array}$ \\
\hline
\end{tabular}


Tabla 2. Principales características de los estudios incluidos (cont.)

\begin{tabular}{|c|c|c|c|}
\hline Autor, año & $\begin{array}{l}\text { Duración } \\
\text { del estudio }\end{array}$ & $\begin{array}{l}\text { Tipo de estudio } \\
\text { Muestra (grupos) }\end{array}$ & Resultados \\
\hline $\begin{array}{l}\text { Talebi M, et al., } \\
2018^{11}\end{array}$ & - & $\begin{array}{c}\text { Estudio de casos y controles } \\
n=45 \\
\text { G1: } 15 \rightarrow \text { usan CPAP } \\
\text { G2: } 15 \rightarrow \text { usan CPAP + terapia cognitivo- } \\
\text { conductual } \\
\text { G3: } 15 \rightarrow \text { sin intervención }\end{array}$ & $\begin{array}{l}\mathrm{G} 1 \rightarrow \text { Resultados positivos en el funcionamiento cognitivo de la } \\
\text { persona y en su calidad de vida } \\
\mathrm{G} 2 \rightarrow \text { Mayores beneficios reportados que G1 en funcionamiento } \\
\text { cognitivo de la persona y calidad de vida }\end{array}$ \\
\hline $\begin{array}{l}\text { Kataoka H, et al., } \\
2019^{12}\end{array}$ & - & $\begin{array}{l}\text { Estudio transversal } \\
\mathrm{n}=1173 \rightarrow \text { tratados con CPAP }\end{array}$ & $\begin{array}{l}\text { Hombres de 30-39 años; } 70-79 \text { años y mujeres de } 50-59 \text { años } \rightarrow \\
\text { EQ-5D con puntuaciones más bajas en pacientes con LS y AOS } \\
\text { que en pacientes sin LS }\end{array}$ \\
\hline $\begin{array}{l}\text { Bani-Issa W, et } \\
\text { al., } 2018^{13}\end{array}$ & - & $\begin{array}{l}\text { Estudio transversal } \\
\qquad n=268\end{array}$ & $\begin{array}{l}\text { Aquellos pacientes que dormían mal son los que peor puntuación } \\
\text { obtenían en CVRS }\end{array}$ \\
\hline $\begin{array}{l}\text { McMillan A, et } \\
\text { al., } 2014^{14}\end{array}$ & 12 meses & $\begin{array}{l}\text { Ensayo aleatorio multicéntrico } \\
\text { G1: BSC (higiene del sueño, siestas, cafeína, } \\
\text { pérdida de peso) }+C P A P \rightarrow n=140 \\
\text { G2: solo } B S C \rightarrow n=138\end{array}$ & $\begin{array}{l}\text { El dominio energía (vitalidad del SF-36) mejoró a los } 3 \text { meses y a los } \\
12 \text { meses }\end{array}$ \\
\hline $\begin{array}{l}\text { Addison-Brown } \\
\text { KJ, et al., 2014 }\end{array}$ & - & $\begin{array}{l}\text { Estudio transversal } \\
\qquad n=2925\end{array}$ & $\begin{array}{l}\text { Efectos más notorios en mediana edad. A partir de los } 70 \text { años } \\
\text { efectos más atenuados } \\
\text { Menor calidad de vida tanto en componentes mentales como físicos }\end{array}$ \\
\hline $\begin{array}{l}\text { Tao W, et al., } \\
2015^{7}\end{array}$ & - & $\begin{array}{c}\text { Revisión bibliográfica } \\
49 \text { estudios } \rightarrow \text { finalmente se utilizan } 5 \text { artículos }\end{array}$ & $\begin{array}{l}\text { Tras el uso del CPAP: mayor calidad de vida } \\
\text { Mejora síntomas AOS, especialmente somnolencia diurna }\end{array}$ \\
\hline $\begin{array}{l}\text { Crawford-Achour } \\
\text { E, et al., } 2015^{15}\end{array}$ & 12 meses & $\begin{array}{c}\text { Estudio de cohortes } \\
n=126 \rightarrow 26 \% \text { usaban CPAP }\end{array}$ & $\begin{array}{l}126 \text { pacientes mayores de } 65 \text { años } \rightarrow 66 \% \text { mostró mejoría en su } \\
\text { calidad de vida }\end{array}$ \\
\hline $\begin{array}{l}\text { Parra 0, et al., } \\
2015^{19 .}\end{array}$ & 5 años & $\begin{array}{l}\text { Estudio prospectivo, aleatorizado, controlado } \\
\qquad \text { y multicéntrico } \\
2 \text { grupos: } \\
\text { G1: uso de CPAP: } 71 \\
\text { G2: no CPAP: } 69\end{array}$ & $\begin{array}{l}\text { En lo que respecta a la calidad de vida, ni en los componentes físicos } \\
\text { ni en los mentales existe mejora significativa } \\
\text { CPAP mejora la supervivencia cardiovascular a largo plazo en } \\
\text { pacientes con ACV y AOS moderada-grave }\end{array}$ \\
\hline $\begin{array}{l}\text { McMillan A, et } \\
\text { al., } 2015^{20}\end{array}$ & 27 meses & $\begin{array}{l}\text { Ensayo multicéntrico, aleatorizado, paralelo } \\
\text { y cegador para investigadores } \\
\qquad n=231 \\
\text { G1: CPAP + cuidados de soporte } \\
\text { G2: solo cuidados de soporte }\end{array}$ & $\begin{array}{l}\text { Grupo 1: reducen la somnolencia diurna, mejora la calidad de vida y } \\
\text { es más rentable que BSC durante } 12 \text { meses }\end{array}$ \\
\hline
\end{tabular}

ACV: accidente cerebrovascular; AOS: apnea obstructiva del sueño; BSC: cuidados de soporte; CPAP: continuous positive airway pressure; CVRS: calidad de vida relacionada con la salud; DM2: diabetes mellitus tipo 2; EQ-5D: EuroQ0I-5D; G1: grupo 1; G2: grupo 2; LS: síndrome locomotor; SF-36: Medical outcomes survey short-form general health survey.

terísticas de los estudios seleccionados (autor/es y año de publicación, duración del estudio, tipo de estudio, tamaño de la muestra, grupos que forman el caso, si procede, y principales resultados), en los cuales se han recolectado datos referentes a la calidad de vida de las personas de edad avanzada con apnea obstructiva del sueńo y los diferentes tratamientos que existen para paliar este síndrome y aumentar por lo tanto la calidad de vida de este grupo de población.

\section{Resultados sobre la relación apnea obstructiva del sueño y calidad de vida en personas de edad avanzada}

Algunos investigadores afirman encontrar relación entre apnea obstructiva del sueńo y calidad de vida de las personas de edad avanzada ${ }^{5-15}$.

Debido a que la mayoría de los pacientes con apnea del sueńo presentan alguna comorbilidad, se hace especialmente relevante saber cómo será la evolución de estas y cómo influirá en la calidad de vida de las personas. De esta forma, el estudio de Bani-Issa et al. ${ }^{13}$, que investiga la relación entre calidad del sueño y calidad de vida de personas con diabetes mellitus tipo 2 mayores de 65 ańos, encuentra que existe relación entre estas variables, especialmente en los dominios "Percepción subjetiva de calidad del sueño", "Uso de medicación para dormir" y" Funcionamiento diurno deteriorado" de la escala $\mathrm{HRQOL}^{13}$. En lo que se refiere a deterioro cognitivo, los autores Petrousky et al. ${ }^{6}$ y Talebi et al. ${ }^{11}$ concluyen en la relación entre esta comorbilidad y la calidad de vida de las personas de edad avanzada con apnea del sueńo. Kataoka et al. ${ }^{12}$ investigan a personas con síndrome locomotor y apnea del sueño cuando son mayores de 65 años, y encuentran que están estrechamente relacionados, especialmente para hombres en rango de edad 30-39 y 70-79 años, y en mujeres para el rango de edad 50-59 años ${ }^{12}$. 
La CPAP o presión positiva continua en la vía aérea ha mostrado efectos beneficiosos sobre la calidad de vida de personas de edad avanzada, disminuyendo el riesgo de caídas, los accidentes cerebrovasculares y la mortalidad cardiovascular, entre otros; así lo corroboran diversos

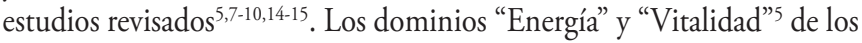
cuestionarios que evalúan la calidad de vida son los que obtienen mayor puntuación tras el uso de la CPAP.

\section{Resultados sobre la no relación entre apnea obstructiva del sueño y calidad de vida en personas de edad avanzada}

Por el contrario, existen autores que en sus investigaciones no han encontrado dicha asociación ${ }^{16-20}$.

La mayoría de estos estudios afirman que, a partir de determinada edad, 70 ańos aproximadamente, se produce un efecto protector, y el SAOS no provoca alteraciones significativas sobre su calidad de vida ${ }^{17,18}$. Aun así, es recomendable el uso de la CPAP para atenuar el resto de síntomas propios de la enfermedad, entre los que destacan: la mejora de la somnolencia diurna ${ }^{7,20}$, el mejor funcionamiento $\operatorname{cognitivo~}^{11}$ y el aumento de supervivencia cardiovascular a largo plazo en pacientes con apnea del sueńo en grado moderado-grave ${ }^{19}$.

El uso de la CPAP es más rentable en el rango de edad 65-70 años, usándola durante 12 meses, que solamente los cuidados de soporte, que consisten en consejos dietéticos, posturales y educación para la salud ${ }^{20}$.

\section{- DISCUSIÓN}

El concepto "calidad de vida" surge cuando las necesidades primarias básicas han quedado satisfechas con los recursos de que disponemos. Incrementar la calidad de vida en personas mayores de 65 años con apnea del sueńo conlleva que exista un aumento de su cuidado de forma independiente, se reduzcan las complicaciones asociadas a las comorbilidades de la persona y disminuya el consumo de recursos sanitarios ${ }^{1}$.

La apnea obstructiva del sueño produce, en personas ancianas, según la mayoría de los estudios revisados ${ }^{5-15}$, un deterioro de la calidad de vida, aunque existe disparidad en los resultados, ya que ciertas investigaciones ${ }^{17,18}$ afirman que a partir de cierta edad (70 ańos aproximadamente) se produce una estabilización en la calidad de vida de las personas que padecen apnea del sueño.
Comparar estos estudios resulta difícil, ya que los instrumentos (SF$36^{8,14,16,17,19}$, SAQLI $^{8,14}$, QSQ ${ }^{9,10}$, EuroQoL-5D ${ }^{12,20}$, HRQOL $^{13}$ y SF-12 ${ }^{18}$ ) y/o metodologías (casos y controles ${ }^{9,11}$, cohortes ${ }^{10,15,17}$, revisión sistemática $^{5-7}$, estudio transversal ${ }^{12,13,16,18}$, prospectivo ${ }^{8,19}$ ) utilizados son distintos en muchos de ellos, al igual que las posibles comorbilidades asociadas (insuficiencia cardiaca ${ }^{5,9,16}$, hipertensión ${ }^{5,16,17}$, accidente cerebrovascular ${ }^{5,9,11}$, diabetes tipo $2^{5,17}$, síndrome locomotor ${ }^{12}$, etc.) a cada individuo.

En la actualidad no existe tratamiento curativo de forma definitiva de la apnea obstructiva del sueńo; solo la CPAP es un dispositivo que, junto a determinadas terapias complementarias, como consejo dietético o educación para la salud, ha reportado ciertas mejoras en determinados aspectos de la calidad de vida de la persona.

Los estudios que investigan a personas de mediana edad concluyen que estas obtienen mayor número de beneficios cuando usan CPAP en los dominios físicos y mentales que las personas de edad avanzada ${ }^{21}$.

Sería recomendable, por lo tanto, la validación de herramientas que evalúen la calidad de vida en la franja de edad de 65 ańos o más, ya que así se podrá tener un enfoque más personalizado y un tratamiento más adecuado.

En definitiva y como consecuencia de la tendencia creciente de personas de edad avanzada en España, se hace necesaria una política sanitaria adecuada, la cual invierta sus esfuerzos en ofrecer mecanismos para que estas personas sean atendidas de forma adecuada y justa, con el principal objetivo de que el aumento de años vividos se haga con la mejor calidad de vida posible ${ }^{1}$.

\section{- CONCLUSIONES}

Padecer apnea obstructiva del sueño en la edad avanzada no es indicativo de una mala calidad de vida del usuario. Existen estudios que avalan dicha relación, a la par que otros muestran lo contrario.

En la actualidad, solo el uso de la CPAP muestra cierta mejoría significativa en algunos de los dominios de la calidad de vida de personas de edad avanzada que padecen apnea obstructiva del sueńo. El resto de tratamientos no han mostrado eficacia en lo que a calidad de vida se refiere.

Se necesitan estudios especializados en este grupo de población

\section{Conflicto de intereses}

La autora declara no tener ningún conflicto de intereses relacionado con este artículo.

\section{BIBLIOGRAFÍA}

1. Serrano Merino J, Pérula-de-Torres LA (dir), Jurado Gámez B (dir Calidad de vida en mayores de 65 años enfermos de SAHS en tratamiento con CPAP. [Tesis doctoral en internet]. [Córdoba]: Universidad de Córdoba;2018 [citado 21 de agosto de 2019]. Recuperado a partir de: https://helvia.uco.es/xmlui/handle/10396/16962

2. Grupo Español de sueño (GES). Consenso Nacional sobre el síndrome de apneas-hipopneas del sueño. Definición y concepto, fisiopatología, clínica y exploración del SAHS. Arch Bronconeumol. 2015;41:12-29.

3. Lloberes P, Durán-Cantolla J, Martínez-García MA, Marín JM, Ferrer A, Corral J, et al. Diagnóstico y tratamiento del síndrome de apneas-hipopneas del sueño. Normativa SEPAR. Arch Bronconeumol. 2011;47(3):143-56.

4. Del-Pino-Casado, R. Cómo aprender (y enseñar) a realizar búsquedas en CINHAL y PubMed. Eventia. 2017:14:1-11.

5. Serrano Merino J, Pérula-de-Torres LA, Muñoz Gómez R, Roldán Villalobo A, Feu Collado MN, Ruíz-Moral R, et al. Impact of CPAP therapy on health-related quality of life in elderly patients with apnoea-hypopnea syndrome: a systematic review of randomised clinical trials. Eur Respir J. 2017;49(1):1601644.
6. Petrousky DV, McPhilips MV, Li J, Brody A, Caffeé L, Hodgson NA Sleep disruption and quality of life in persons with dementia: $A$ state-of-the-art review. Geriatr Nurs. 2018:39:640-5.

7. Wang T, Huang W, Zong H, Zhang Y. The efficacy of continuous positive airway pressure therapy on nocturia in patients with obstructive sleep apnea: A systematic review and meta-analysis. Int $\mathrm{J}$ Neurourol. 2015:19(3):178-84.

8. Serrano Merino J, Pérula-de-Torres LA, Bardwell WA, Muñoz Gó mez R, Roldán Villalobos A, Feu Collado N, et al. Impact of Positive Pressure Treatment of the Airway on Health-Related Quality of Life in Elderly Patients With Obstructive Sleep Apnea. Biol Res Nurs. 2018;20(4):452-61

9. Dalmases M, Sole-Padulles C, Bartres-Faz D, Nunez L, Embud C, Torres M, et al. Obstructive sleep apnea in elderly patients: neurocognitive function before and after CPAP treatment. Am J Respir Crit Care Med. 2014;189:A2418.

10. Martínez-García MA, Chiner E, Hernández L, Cortés JP, Catalán P, Ponce $S$, et al. Obstructive sleep apnea in the elderly: role of continuous positive airway pressure treatment. Eur Respir J. 2015;46(1):142-51.
11. Talebi M, Tabatabaye KR, Vahedi E. Application of cognitive-behavioral therapy in obstructive sleep apnea: comparison of combined treatment, and CPAP theraphy on executive functions of the brain. Sleep Hypn. 2018;20(3):148-59

12. Kataoka M, Miyatake N, Ichikawa $H$, Arakawa $Y$, Mori $Y$. Sub-analysis of the prevalence of locomotive syndrome and its relationship with health-related quality of life in patients with obstructive sleep apnea syndrome as classified by age and sex. Sleep and biological rhythms. 2019;17(1):149-53.

13. Bani-Issa W, Al-Shujairi AM, Patrick L. Association between quality of sleep and health-related quality of life in persons with diabetes mellitus type 2. J Clin Nurs. 2018;27(7-8):1653-61.

14. McMillan A, Bratton D, Faria R, Laskawiec-Szkonter M, Griffin $\mathrm{S}$, Davies RJ, et al. Continuous positive airway pressure in older people with obstructive sleep apnea syndrome (PREDICT): a 12 months, multicentre, randomised trial. Lancet Respir. 2014; 2(10):804-12.

15. Crawford-Achour E, Dauphirot V, Saint Martin M, Tardy M, Gonthier R, Barthelemy JC, et al. Protective effect of long-term CPAP 
Lucía Ortega Donaire

Calidad de vida en personas mayores con síndrome de apnea obstructiva del sueño. Revisión sistemática

therapy on cognitive performance in elderly patients with severe OSA: The proof study. J Clin Sleep Med. 2015;11(5):519-24.

16. Lima T, Brito EC, Martins R, Lima SG, Pedrosa RP. Obstructive sleep apnea and quality of life in elderly patients with a pacemaker. J Bras Pneumol. 2019;45(1):e20170333.

17. Appleton SL, Vakulin A, McEvoy RD, Vincent A, Martin SA, Grant JF, et al. Undiagnosed obstructive sleep apnea is independently associated with reductions in quality of life in middle-aged, but not elderly men of a population cohort. Sleep Breath. 2015;19(4):1309-16.
18. Addison-Brown KJ, Letter AJ, Yaggi K, McClure LA, Unverzagt F, Howard VJ, et al. Age differences in the association of obstructive sleep apnea risk with cognition and quality of life. J Sleep Res. 2014:23(1):69-79

19. Parra O, Sánchez A, Capote E, Bonnin M, Arboix A, Campos-Rodríguez $\mathrm{F}$, et al. Efficacy of continuous positive airway pressure treatment on 5- year survival in patients with ischaemic stroke and obstructive sleep apnea: a randomized controlled trial. J Sleep Res. 2015;24:47-53
20. McMillan A, Bratton DJ, Faria R, laskawiec-Skonter M, Griffin S Davies RJ, et al. A multicentre randomised controlled trial and economic evaluation of continuous positive airway pressure for the treatment of obstructive sleep apnea syndrome in older people. PREDICT. Health Technol Assess. 2015:19(40):1-188.

21. Lee W, Lee SA, Ryu HU. Ouality of life in patients with obstructive sleep apnea: Relationship with daytime sleepiness, sleep quality, depression, and apnea severity. Chron Respir Dis. 2016;13(1):33-9 